\title{
ANH
}

\section{PRÁticas de incentivo e APOIO À AMAMENTAÇÃo de RECÉM- NASCIDOS PREMATUROS NA PERSPECTIVA DA MÃE}

\author{
Ana Leticia Monteiro Gomes ${ }^{1}$, Talita Balaminut ${ }^{2}$, Bruna Nunes Magesti ${ }^{3}$, Danielle Lemos Querido ${ }^{4}$,
} Carmen Gracinda Silvan Scochi ${ }^{5}$, Marialda Moreira Christoffel ${ }^{6}$

\section{RESUMO}

Objetivo: Analisar as práticas de incentivo e apoio à amamentação de recém-nascidos prematuros no âmbito hospitalar e logo após a alta hospitalar, na perspectiva da mãe.

Métodos: Estudo descritivo realizado no ambulatório de follow-up de um Hospital Amigo da Criança do Município do Rio de Janeiro, nos meses de junho a outubro de 2015. A coleta de dados foi realizada por meio de entrevista, com um formulário estruturado, com 17 mães de 21 recém-nascidos prematuros, nas duas primeiras semanas após a alta hospitalar. Os dados foram organizados a partir do software Excel $®$, exportados para o programa estatístico $R \circledast$ e analisados utilizando estatística descritiva.

Resultados: No âmbito hospitalar, $17,6 \%$ das mães realizaram o contato pele a pele na sala de parto, $94,1 \%$ realizaram ordenha mamária, 94,1\% amamentaram diretamente no seio materno e 58,8\% das mães participaram dos grupos de apoio durante o pré-natal e/ou durante a internação. Após a alta hospitalar, 93,7\% das mães receberam ajuda do profissional de saúde para amamentar.

Conclusão: É necessário o fortalecimento de ferramentas que sustentem as estratégias de apoio ao aleitamento materno para o início e manutenção da amamentação durante e após a internação hospitalar.

Descritores: Aleitamento materno; Mães; Recém-nascido Prematuro; Enfermagem neonatal; Promoção da saúde.

1. Ana Leticia Monteiro Gomes - Enfermeira. Doutora em Enfermagem. Universidade Federal do Rio de Janeiro/Escola de Enfermagem Anna Nery (UFRJ/ EEAN). Professora Adjunta. Rio de Janeiro, RJ, Brasil. E-mail: analeticiagomes88@gmail.com ORCID iD: 0000-0001-6220-5261

2. Talita Balaminut - Enfermeira. Doutora em Enfermagem. Universidade de São Paulo/ Escola de Enfermagem de Ribeirão Preto (USP/ EERP). Ribeirão Preto, São Paulo, Brasil. E-mail: talita_balaminut@yahoo.com.br ORCID iD: 0000-0002-9746-3102

3. Bruna Nunes Magesti - Enfermeira. Mestra em Enfermagem. Universidade Federal do Rio de Janeiro/Escola de Enfermagem Anna Nery (UFRJ/EEAN). Rio de Janeiro, RJ, Brasil. E-mail. ORCID iD:0000-0001-9901-6659

4. Danielle Lemos Querido - Enfermeira. Mestra em Enfermagem. Mestra em Enfermagem. Universidade Federal do Rio de Janeiro/Escola de Enfermagem Anna Nery (UFRJ/EEAN). Rio de Janeiro, RJ, Brasil. E-mail. danyquerido@me.ufrj.br ORCID iD: 0000-0003-4895-296X

5. Carmen Gracinda Silvan Scochi - Enfermeira. Enfermeira. Doutora em Enfermagem. Universidade de São Paulo/ Escola de Enfermagem de Ribeirão Preto (USP/ EERP). Professora Titular. Ribeirão Preto, São Paulo, Brasil. E-mail: carmenscochi@gmail.com ORCID iD: 0000-0001-5865-9711

6. Marialda Moreira Christoffel - Enfermeira. Pós-doutorado em Enfermagem. Universidade Federal do Rio de Janeiro/Escola de Enfermagem Anna Nery (UFRJ/EEAN). Professora Associada. Rio de Janeiro, RJ, Brasil. E-mail: marialda.ufrj@gmail.com. ORCID iD: 0000-0002-4037-8759

\section{Artigo Original}

\section{Autor Correspondente:}

Ana Leticia Monteiro Gomes.

Endereço completo: Rua Afonso Cavalcanti, 275.

Data de submissão: $17 / 06 / 2019$

Cidade Nova. CEP: 20211-110.

Rio de Janeiro, RJ, Brasil.

Telefones: (21) 2293-8999 e E-mail: analeticiagomes88@gmail

Como citar esse artigo

GOMES, A. L. M. et al. Práticas de incentivo e apoio à amamentação de recém-nascidos prematuros na perspectiva da mãe.

Advances in Nursing and Health, v. 1, p. 98-112, Londrina, 2019. 


\section{INTRODUÇÃO}

O aleitamento materno é uma das principais estratégias para a redução da morbimortalidade em recém-nascidos prematuros (RNPTs). Essa é uma iniciativa importante para a sobrevida dos RNPTs, cujo impacto é positivo tanto sobre o tempo de internação, prognóstico, crescimento e desenvolvimento neurológico, como quanto ao impacto econômico[1].

No entanto, apesar dos benefícios supracitados, a prevalência de aleitamento materno em RNPTs é menor comparada aos recém-nascidos a termo[2], sendo necessário discutir as estratégias de apoio utilizadas para iniciar e manter a amamentação dos RNPTs a fim de aumentar essa prevalência.

Dentre as estratégias adotadas para aumentar a prevalência da prática da amamentação em RNPTs estão as ações de apoio ao aleitamento materno. O estímulo ao contato pele a pele, que se inicia com o acoIhimento da família na unidade neonatal, o aconselhamento em amamentação no pré-natal e pós-natal, programas educativos para profissionais de saúde e familiares são exemplos dessas estratégias de apoio.
Para promover e apoiar a prática da amamentação do RNPT, em 2012, um grupo de pesquisadores dos países nórdicos e do Canadá elaboraram a expansão da Iniciativa Hospital Amigo da Criança para Unidades Neonatais (IHAC-Neo), levando em consideração as necessidades inerentes aos prematuros, já que precisam de um cuidado diferenciado em relação à amamentação no contexto da unidade neonatal[3].

A IHAC-Neo apresenta três princípios: a atitude da equipe, que deve focar e responder às necessidades individuais de cada mãe no seu contexto; o incentivo às ações facilitadoras da abordagem do cuidado centrado na família; e, por fim, a continuidade do cuidado entre os períodos pré, peri e pós-natal, bem como após a alta hospitalar[3].

A IHAC-Neo também propõe Dez Passos para o Sucesso do Aleitamento Materno Exclusivo em Unidades Neonatais: 1. Ter uma política escrita que é rotineiramente comunicada para todos os profissionais de saúde; 2. Educar e treinar todos os profissionais de saúde em conhecimentos específicos e nas habilidades necessárias para implementar essa política; 3. Informar todas as gestantes hospitalizadas com risco de nascimento prematuro ou criança doente sobre o manejo 
da lactação e amamentação, e os benefícios do aleitamento materno; 4. Encorajar precocemente o contato pele a pele mãe-bebê de maneira contínua e prolongada (Cuidado Canguru); 5. Demonstrar às mães como iniciar e manter a lactação e estabelecer a estabilidade do bebê como único critério para o início precoce da amamentação; 6 . Não oferecer alimentos ou outras bebidas que não sejam leite humano aos recém-nascidos, exceto se houver indicação médica; 7. Permitir que mães e bebês permaneçam juntos 24 horas por dia; 8. Encorajar livre demanda ou, quando necessário, a semidemanda de amamentação como uma estratégia transicional para prematuros ou bebês doentes; 9. Uso de métodos alternativos à mamadeira pelo menos até o completo estabelecimento da amamentação e somente uso de bicos ou chupetas por motivos justificados; 10. Preparar os pais para a continuidade do aleitamento materno e assegurar acesso a grupos/ serviços de apoio após a alta hospitalar[3].

\section{O processo de amamentar o prematuro} não é fácil, pois as mães de RNPTs enfrentam uma situação extremamente estressante no momento do nascimento, onde ocorre a separação mãe-bebê. Além disso, ainda não há consenso sobre o momento exato para o RNPT iniciar a amamentação (sucção no seio materno). Estudo sugere a estabilidade clínica como único critério para iniciar a amamentação, sem a necessidade de programas de estimulação oral[3]. Já, outros autores sugerem aos profissionais de saúde a aplicação da estimulação do sistema sensório-motor-oral sensório-motor oral, para promover o desenvolvimento das funções orais de neonatos antes de iniciar a amamentação[4].

Devido os desafios supracitados, muitas mães referem dificuldades relacionadas ao início e manutenção do aleitamento materno, tais como a progressão lenta da amamentação e exaustão materna devido ao número elevado de ordenhas mamárias necessárias para a manutenção da lactação/ amamentação[5]. Além disso, no contexto da prematuridade, a amamentação torna-se uma questão secundária no meio dos medos da perda, da angústia do tempo de separação, devido à internação prolongada e à instabilidade clínica do seu filho[6].

Diante do exposto, o objetivo deste estudo foi analisar as práticas de incentivo e apoio à amamentação de recém-nascidos prematuros no âmbito hospitalar e logo após a alta hospitalar, na perspectiva da mãe. 


\section{MÉTODO}

O presente estudo faz parte da pesquisa multicêntrica intitulada "Aleitamento materno em prematuros: impacto da IHAC para unidades neonatais". Trata-se de um estudo descritivo realizado em ambulatório de follow-up de uma maternidade pública, com título de Hospital Amigo da Criança, localizada no Município do Rio de Janeiro (hospital controle da Região Sudeste do Brasil).

O cálculo amostral para a coleta de dados deste estudo teve como base o cálculo realizado para o estudo multicêntrico, utilizando o programa de computador denominado G Power analysis 3.1., obtendo-se um tamanho amostral mínimo de 21 mães de prematuros para cada hospital. Desse modo, o presente estudo aconteceu com 17 mães de 21 recém-nascidos pré-termo (quatro gêmeos) atendidos nas duas primeiras semanas após a alta hospitalar, nos meses de junho a outubro de 2015.

Foram incluídas no estudo as mães de recém-nascidos prematuros que ficaram internados na unidade de terapia intensiva neonatal da instituição pesquisada, nas primeiras 48 horas de nascimento, que frequentaram o ambulatório no período de coleta de dados. Foram excluídas as mães que tinham histórico de distúrbios psiquiátricos e/ou problemas neurológicos e as que tinham contraindicação temporária ou definitiva para o aleitamento materno (exemplo: ser portadora do vírus da imunodeficiência humana e/ou vírus T-linfotrópico humano 1, ou estar em uso de medicamentos que impediam a amamentação).

Os dados foram coletados a partir de uma entrevista individual guiada por um instrumento estruturado onde eram anotadas as respostas das participantes do estudo. A duração média de cada entrevista foi de 15 minutos. Antes da coleta de dados, realizou-se um teste piloto com cinco mães, sendo duas com filhos gêmeos, perfazendo um total de sete prematuros que não foram incluídos na amostragem final. A partir dos resultados encontrados no teste piloto, o instrumento de coleta de dados foi ajustado quanto ao seu conteúdo.

O instrumento de coleta de dados continha cinco blocos: dados sociodemográficos das mães, dados gestacionais e do parto, dados do nascimento, condições do RNPT durante a internação e dados sobre as estratégias de apoio ao aleitamento materno (acolhimento dos pais na UTIN, realização 
do contato pele a pele e/ou posição canguru, ordenha mamária, oferecimento de orientações sobre o aleitamento materno, participação em grupos de apoio para amamentação e recebimento de materiais educativos sobre o aleitamento materno).

Para a realização da coleta de dados, a pesquisadora ou o auxiliar de pesquisa compareciam ao ambulatório nos dias marcados e, em uma sala reservada, realizavam a leitura do Termo de Consentimento Livre e Esclarecido e coletavam as assinaturas das participantes; a seguir, iniciavam a entrevista.

Os dados obtidos foram duplamente digitados e organizados em planilhas, com auxílio do software Excel $®$ versão 2010, e comparados para correção das inconsistências. Posteriormente foram exportados para o programa estatístico $R \circledast$ versão 3.1.1.

Após essa etapa, os dados foram analisados de forma descritiva, a partir de porcentagem simples, média $(\overline{\boldsymbol{x}})$ e apresentados em tabelas.

Em consonância aos preceitos éticos em pesquisas que envolvem seres humanos, da Resolução no 466, de 12 de dezembro de 2012, a pesquisa obteve parecer favorável pelo Comitê de Ética em Pesquisa com protocolo CAAE: 44033215.3 .0000 .5238 e Parecer número: 1.349.465.

\section{RESULTADO}

Este estudo contou com maior participação de mulheres na faixa etária de 30 a 41 anos (52,9\%) e com o nível de escolaridade ensino médio completo $(41,1 \%)$. Quanto às informações relacionadas à gestação e parto, $58,8 \%$ (10) das mulheres afirmaram ter vivenciado partos anteriores e, dessas, $20 \%$ (2) afirmaram que tiveram parto prematuro e $90 \%$ (9) das mães haviam amamentado (seio materno) seus recém-nascidos pelo menos alguma vez. Em relação à gestação mais atual, 88,2\% (15) das mães receberam orientações sobre aleitamento materno durante o pré-natal, 88,2\% (15) tiveram parto cesáreo e apenas $17,3 \%$ (3) alegaram ter realizado contato pele a pele com o RNPT no momento do nascimento. Nenhum destes RNPTs foi amamentado na primeira hora de vida e $66,6 \%$ (14) tiveram desconforto respiratório no momento do nascimento, assim como $47,6 \%$ (10) dos RNPTs necessitaram de reanimação neonatal; 61,9\% (13) foram prematuros tardios (32 até 36 semanas), 


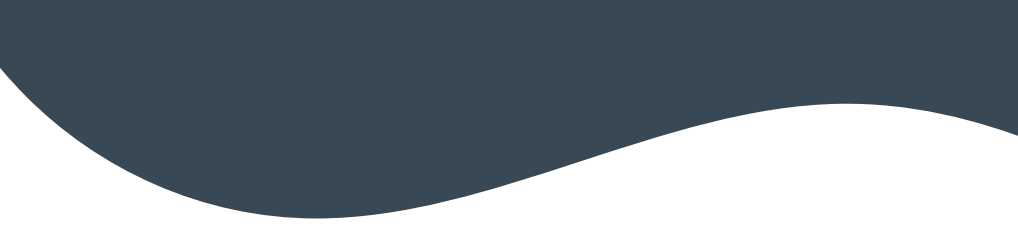

$76,1 \%$ (16) tiveram baixo peso e 9,52\% (2)

tiveram o Apgar do 50 minuto menor que 7.

A distribuição da frequência com que aconte- ceram as estratégias de apoio inseridas nos passos 3, 4, 5, 7 da IHAC-Neo está descrita na Tabela 1, a seguir.

Tabela 1 - Distribuição das estratégias de apoio para o início e manutenção da amamentação na perspectiva de mães de RNPTs $(n=17)$, no ambulatório de follow-up.

Rio de Janeiro/RJ, Brasil, 2015.

VARIÁVEIS

ACOLHIMENTO DOS PAIS NA UTIN NO PRIMEIRO DIA DO NASCIMENTO

Mãe

Pai

APROXIMAÇÃO FÍSICA DA MÃE COM O PREMATURO POR CONTATO PELE A PELE NO TÓRAX MATERNO

Logo após o nascimento

3

$>24^{\mathrm{a}}$ hora

Não houve

APROXIMAÇÃO FÍSICA DA MÃE COM O PREMATURO POR CONTATO PELE A PELE PELO TOQUE

Logo após o nascimento

4

Entre a $2^{\mathrm{a}}$ e a $24^{\mathrm{a}}$ hora

$>24^{\mathrm{a}}$ hora

REALIZAÇÃO DA POSIÇÃO CANGURU NA INTERNAÇÃO

Mãe

Pai*

15
$\mathbf{N}$

$\%$ 17,6

82,3

,6


REALIZAÇÃO DA POSIÇÃO CANGURU NO DOMICÍLIO

Mãe

12

70,6

Pai*

7

41,2

ORIENTAÇÃO SOBRE ORDENHA NA

$1^{\text {a }}$ VISITA DA MÃE À UTIN

Sim

8

47,1

Não

8

47,1

Ignorado

1

5,9

REALIZAÇÃO DA ORDENHA NA INTERNAÇÃO

Sim

16

94,1

Não

1

5,9

ORIENTAÇÃO SOBRE AMAMENTAÇÃO

DURANTE A INTERNAÇÃO

Sim

16

94,1

Não

1

5,9

FREQUÊNCIA DE ORIENTAÇÕES SOBRE

AMAMENTAÇÃO DURANTE A INTERNAÇÃO

Diariamente

7

43,8

Na maior parte do tempo

5

31,2

Somente nos primeiros dias

4

25,0

PARTICIPAÇÃO EM GRUPOS DE APOIO

PARA AMAMENTAÇÃO

Sim

10

58,8

Não

7

41,1 
RECEBIMENTO DE MATERIAL EDUCATIVO SOBRE OS BENEFÍCIOS DA AMAMENTAÇÃO

Sim

Não

*Dados fornecidos pela mãe.

Cabe ressaltar que três (14,3\%) RNPTs receberam contato pele a pele no tórax materno imediatamente após o nascimento. Tais RNPTs nasceram com idade gestacional, pela data da última menstruação (DUM), de 33 semanas e 2 dias (dois RNPTs) e 32 semanas e 5 dias (um RNPT).

Em relação ao contato pele a pele na posição canguru (passo 4 da IHAC-Neo), $80,9 \%$ (17) dos RNPTs tiveram esse contato em algum momento da internação. Desses, $64,7 \%$ (11) experimentaram a posição canguru com mais de 24 horas de vida e para $28,5 \%$ (6) as mães dos RNPTs não se lembravam do momento correto em que a realizaram. A média de dias para a realização da primeira posição canguru, das mães que se lembravam da informação, foi de 10,4 ( \pm 6,9) dias. Já, em relação a essa prática no domicílio, somente $70,6 \%$ dos prematuros continuaram a receber contato pele a pele por meio da posição canguru.

Em relação ao passo 5 da IHAC-Neo, a prática da ordenha mamária, 12,5\% (2) das mães a realizaram nas primeiras 24 horas de vida do RNPT; 23,5\% (4), entre 48 e 72 horas de vida; 52,9\% (9), depois de 72 horas de vida; e 5,8\% (1) não se lembravam da informação. Quanto ao primeiro local de realização, para $50 \%$ foi no banco de leite; $43,75 \%$, na unidade de terapia intensiva neonatal (UTIN); e 6,25\% não se lembravam do local. Além disso, $87,50 \%$ das 16 mães que retiraram leite tiveram ajuda da equipe de enfermagem nesta técnica.

Sobre o momento em que os grupos de apoio para amamentação eram oferecidos às mães, 60\% (6) informaram participação durante o pré-natal (passo 3 da IHAC-Neo); $20,0 \%$ (2), durante o pré-natal e a sua inter- 
nação (passos 3 e 5 da IHAC-Neo); 10\% (1), depois da sua alta e durante a internação do bebê (passos 5 e 10 da IHAC-Neo); e 10\% (1), apenas durante sua internação na maternidade (passo 5 da IHAC-Neo).

Destaca-se que, durante a internação hospitalar dos RNPTs, 82,3\% (14) das mães receberam ajuda dos profissionais de saúde para amamentar e 100\% (17) sentiram-se apoiadas para amamentar pelo Banco de Leite Humano.

Em relação à ajuda e ao apoio oferecidos às mães pelos profissionais de saúde após a internação hospitalar do RNPT (passo $10), 94,1 \%$ (16) receberam ajuda para amamentar e 52,9\% (9) sentiram-se apoiadas para amamentar.

\section{DISCUSSÃO}

As mães de RNPTs precisam receber estratégias de apoio à amamentação de forma a favorecer o seu início e manutenção. Tais estratégias devem estar baseadas em evidências científicas e devem ser oferecidas de forma sistematizada no pré-natal, durante a internação do bebê na unidade de terapia intensiva neonatal e até após a sua alta, no domicílio[2].
A entrada precoce dos pais na unidade de terapia intensiva neontal (UTIN) e o estímulo à participação destes nos cuidados com o bebê é uma recomendação do Ministério da Saúde e um dos princípios da IHAC-Neo, com a finalidade de promover o vínculo família-bebê, visto que o ambiente da UTIN é frequentemente associado à possibilidade de morte e a vivência desta hospitalização causa um marco na vida familiar[6].

Dentro do que é preconizado pela IHAC-Neo como os Dez Passos para o Sucesso do Aleitamento Materno Exclusivo em Unidades Neonatais, os passos 3 e 5 envolvem o manejo e a manutenção da lactação e, nesse contexto, a ordenha mamária é de extrema relevância.

Para o processo de início e manutenção da lactação e aleitamento materno, conforme é preconizado no $1^{\circ}$ princípio da IHAC-Neo, os profissionais de saúde devem considerar as particularidades que as mães de recém-nascidos internados em unidades neonatais enfrentam, tais como vivenciar o cotidiano hospitalar, lidando com os sentimentos de perda, estresse, ansiedade, adaptação e resiliência[7].

Desse modo, como estratégia de apoio para o início e manutenção do aleitamento 
materno, é necessária a ordenha mamária, que é apontada na literatura científica como uma das práticas que favorece o aleitamento materno. A expressão precoce do leite materno resulta em uma lactação bem-sucedida e por um maior período, nas mães de recém-nascidos de muito baixo peso[8].

O momento da primeira expressão de leite materno está intimamente ligado ao sucesso da lactação. A literatura científica recomenda que os profissionais de saúde encorajem a mãe a estimular a mama e retirar o leite precocemente, de preferência nas primeiras seis horas após o parto(8). Apesar de existir essa recomendação, o presente estudo mostra que essa prática ainda era adiada na unidade onde ocorreu a pesquisa.

Estudo transversal realizado com 100 mães de RNPTs em Milão, na Itália, mostrou que $58 \%$ das mães entrevistadas começaram a retirar o leite materno nas primeiras 24 horas de parto e $12 \%$ dessas iniciaram a ordenha mamária nas primeiras 6 a 8 horas[9]. Tais dados, comparados com os do presente estudo, mostram que o tempo de início da prática da ordenha ainda é um desafio para a instituição pesquisada, pois a maioria (52,9\%) das mães realizou a prática da ordenha após 72 horas do nascimento. A rotina da instituição pesquisada no momento da coleta de dados não estipulava um tempo para início da ordenha e a meta era sempre iniciar a retirada do leite o mais precocemente possível, e todos os profissionais de saúde da instituição poderiam orientar e apoiar a ordenha mamária.

O quarto passo da IHAC-Neo se refere ao contato pele a pele mãe-bebê de forma precoce, de maneira contínua e prolongada. O Método Canguru faz parte de uma política pública de saúde adotada pelo Ministério da Saúde brasileiro para fortalecer o papel da família no cuidado com o RNPT, além de estimular o contato pele a pele de forma precoce. Cabe ressaltar que esse contato pele a pele no momento do nascimento pode ser iniciado pelo toque, até evoluir para a posição canguru quando o RNPT atingir a estabilidade clínica. Desse modo, o contato pele a pele no tórax/abdome materno pode ocorrer no momento do nascimento ou durante a internação na unidade neonatal por meio da posição canguru[10].

A posição canguru é eficaz, devido aos seus benefícios no aumento das taxas de amamentação exclusiva ou quase exclusiva[11]. Na amostra estudada, $17,6 \%$ das mães e $58,8 \%$ dos pais não realizaram a 
posição canguru durante a internação. E a maioria das mães e pais que tiveram a oportunidade de fazer não a realizaram de forma precoce, pois a média do tempo de realização do primeiro contato pele a pele pela posição canguru das mães que se lembravam da informação foi de $10,4( \pm 6,9)$ dias. De acordo com a rotina da instituição, a posição canguru pode ser realizada assim que o RNPT atingir a estabilidade clínica, seja na ocasião do nascimento ou durante a internação na UTIN.

Em relação à prática da posição canguru no domicílio, um estudo transversal realizado com 43 mães de RNPTs atendidos na Unidade de Cuidado Intermediário Neonatal Canguru de um hospital universitário de referência na Região Nordeste do Brasil mostrou que após 15 dias da alta hospitalar a maioria $(90,7 \%)$ das mães ainda fazia uso da posição canguru e que sua duração em $48,7 \%$ das mães era de cerca de 2 a 5 horas por dia. No entanto, apenas 39,5\% das mães receberam a visita domiciliar de seguimento, o que mostra fragilidades no cumprimento da terceira etapa do Método Canguru pela atenção básica e do décimo passo da IHAC-Neo para a maioria das mães atendidas[12].
$\mathrm{Na}$ instituição de saúde referente ao presente estudo, após a alta hospitalar os recém-nascidos prematuros eram encaminhados para o seu seguimento diretamente para as unidades básicas de saúde ou para o ambulatório de follow up da instituição que realiza o cuidado canguru compartilhado com a atenção básica.

Quanto ao apoio à amamentação após a alta hospitalar, a estratégia que está destacada no passo 10 da IHAC-Neo é a maternidade assegurar a manutenção da amamentação por meio do acesso a grupos de apoio. Pesquisadores mostraram que mães de recém-nascidos prematuros tardios que receberam apoio à prática da amamentação durante a internação e que tiveram acesso a informações sobre grupos de suporte à amamentação foram mais propensas a amamentar seus bebê durante os primeiros 10 dias após o nascimento[13].

A participação em grupos de apoio à amamentação, com recebimento de materiais educativos, deve ocorrer desde o pré-natal, perpassando pela internação do RNPT até a alta hospitalar. Infelizmente, os resultados deste estudo evidenciaram que tais estratégias de apoio ao aleitamento mater- 
no não foram oferecidas de forma igualitária para toda a amostra estudada.

É preciso frisar que todos os profissionais de saúde que prestam assistência às mães de RNPTs devem adquirir conhecimentos e as competências necessárias para o manejo da amamentação e as particularidades de se amamentar um prematuro. No entanto, autores de um estudo de revisão integrativa concluíram que os profissionais de saúde, independente da sua área de atuação, precisam ser mais bem capacitados para trabalhar com aleitamento materno, pois a maioria dos estudos utilizados na revisão mostrou que os profissionais de saúde possuíam domínio teórico em relação à amamentação, porém não possuíam domínio prático[14].

O estudo apresentou como limitações o número reduzido de participantes, limitando os resultados encontrados apenas para a amostra em questão, e com perda de duas participantes que não compareceram à consulta marcada no ambulatório de follow-up. Ressalta-se que os dados sobre o tempo de início e duração do contato pele a pele tanto na sala de parto quanto durante a internação foram obtidos por meio da entrevista com as mães, pois não foram encontrados tais registros no prontuário da unidade pesquisada.

\section{CONCLUSÃO}

Os resultados demonstram que as estratégias de apoio para o início e a manutenção do aleitamento materno, como o acolhimento dos pais na UTIN, a ordenha mamária, contato pele a pele, recebimento de orientações e materiais educativos sobre aleitamento materno, participação em grupos de apoio, estão presentes no âmbito hospitalar e domiciliar, mas precisam ser consolidadas e oferecidas a todas as mães de RNPTs internados na unidade de terapia intensiva neonatal.

Durante a internação hospitalar, as estratégias de apoio oferecidas pela instituição pesquisada, na perspectiva das mães, fazem parte do que é preconizado pela IHAC-Neo. Entretanto, algumas estratégias devem ser fortalecidas, tais como as que dizem respeito ao tempo de início da ordenha mamária, que ainda é tardio, assim como ao tempo de início do contato pele a pele no tórax materno no momento do nascimento e à realização da posição canguru durante a internação e a sua continuação no domicílio. Recomenda-se a 
implementação da colostroterapia como rotina na unidade pesquisada, para que, dessa forma, os profissionais de saúde possam estimular a mãe a realizar a ordenha mamária de forma mais precoce. Destaca-se que tal prática também ajuda a promover o contato pele a pele, devido à maior aproximação da mãe com o RNPT.

$E$, em relação à manutenção da amamentação no domicílio, cabe ressaltar que, após a alta hospitalar, houve um decréscimo de mães que se sentiram apoiadas em relação ao aleitamento materno. Tal resultado demonstra fragilidades no cumprimento do passo 10 da IHAC-Neo e os desafios para manutenção da amamentação após a alta hospitalar até os 6 meses, como é preconizado. Portanto, se faz necessário o fortalecimento da articulação do cuidado hospitalar com o cuidado da atenção básica por meio do canguru compartilhado, favorecendo, assim, a criação de grupos de apoio multiprofissionais.

Apoio financeiro: auxílio da agência de fomento Coordenação de Aperfeiçoamento de Ensino Superior (CAPES). Bolsa de Mestrado Acadêmico outubro 2014 a dezembro 2015. 


\section{REFERÊNCIAS}

1. Victora CG, Bahl R, Barros AJ, França GV, Horton S, Krasevec J, et al. Breastfeeding in the 21st century: epidemiology, mechanisms, and lifelong effect. Lancet. 2016 Jan; 387(10017):475-90. doi: 10.1016/S01406736(15)01024-7.

2. Niela-Vilén $\mathrm{H}$, Axelin $\mathrm{A}$, Melender $\mathrm{HL}$, Salantera S. Aiming to be a breastfeeding mother in a neonatal intensive care unit and at home: a thematic analysis of peer support group discussion in social media. Matern Child Nutr. 2015 Oct;11(4):712-26. doi: 10.1111/mcn.

3. Nyqvist $K \mathrm{H}$, Maastrup $\mathrm{R}$, Hansen $M N$, Haggkvist AP, Hannula $L$, Ezeonodo $A$, et al. Neo-BFHI: The baby-friendly Hospital Initiative for Neonatal Wards. Core document with recommended standards and criteria. Nordic and Quebec Working Group [Internet]. 2015 [cited 2019 Jul 27]. Available from: http://epilegothilasmo.gr/wpcontent/uploads/2017/04/Neo_BFHI_Core_ document_2015_Edition.pdf

4. Lemes EF, Silva THMM, correr AMA, Almeida EOC, Luchesi KF. Oral and non-oral sensorimotor stimulation in preterm infants: bibliographic review. Rev CEFAC. 2015 June; 17(3):945-55. doi: 10.1590/1982021620159414

5. Ikonen $\mathrm{R}$, Paavilainen $\mathrm{E}$, Kaunonen $\mathrm{M}$. Preterm infants' mothers' experiences with milk expression and breastfeeding: an integrative review. Adv Neonatal Care. 2015 Dec;15(6):394-406. doi: 10.1097/ ANC. 0000000000000232 .

6. Pereira LB, Abrão ACFV, Ohara CVS, Ribeiro CA. Maternal experiences with specificities of prematurity that hinder breastfeeding. Texto Contexto Enferm. 2015 Mar;24(1):55-63. doi:10.1590/0104-07072015000540014

7. Rossman B, Greene MM, Meier PP. The role of peer support in the development of maternal identity for "NICU Moms". J Obstet Ginecol Neonatal Nurse. 2015Jan-Feb;44(1):3-16. doi: 10.1111/1552-6909.12527

8. Becker GE, Smith HA, Cooney F. Methods of milk expression for lactating women. Cochrane Database Syst Rev. 2016Sept;9(CD006170):1-126. doi: 10.1002/14651858.CD006170.pub5

9. Gianni ML, Bezze EN, Sannino P, Baro M, Roggero $\mathrm{P}$, Muscolo $\mathrm{S}$, et al. Maternal views on facilitators of and barriers to breastfeeding preterm infants. BMC Pediatr. 2018 Dec;18(1):283. doi: 10.1186/s12887-018$1260-2$

10. Ministério da Saúde (BR). Secretaria de Atenção à Saúde. Departamento de Ações Programáticas Estratégicas. Atenção humanizada ao recém-nascido: método canguru, diretrizes do cuidado [Internet]. Brasília DF: Ministério da Saúde; 2018 [cited 2019 jun. 17]. Disponível em: http:// portaldeboaspraticas.iff.fiocruz.br/biblioteca/ metodo-canguru-diretrizes-do-cuidado

11. Conde-Agudelo A, Díaz-Rossello J.L. Kangaroo mother care to reduce morbidity and mortality in low birthweight infants. Cochrane Database Syst Rev. 2016 Aug;8(CD002771):1-137. doi: 10.1002/14651858.CD002771.pub4.

12. Feitosa MR, Gubert FA, Tomé MABG, Pinheiro MTM, Neves CS, Benevides JL, et al. Primary health care follow-up visits: investigation of care continuity of preterm newborns from a kangaroo-mother care unit. Int Arch Med. 2017 Feb;10(32):1-9. doi: 10.3823/2302 
13. Rayfield S, Oakley L, Quigley MA. Association between breastfeeding support and breastfeeding rates in the UK: a comparison of late preterm and term infants. BMJ Open. 2015 Nov;5(11):e009144. doi:10.1136/ bmjopen-2015-009144

14. Almeida JM, Luz SAB, Ued FV. Support of breastfeeding by health professionals: integrative review of the literature. Rev Paul Pediatr. 2015 July-Sept;33(3):355-62. doi: 10.1016/j.rpped.2014.10.002 